\title{
The Oxygen Reduction Reaction on Pt Single Crystal Electrodes Modified with Aromatic Organic Molecules
}

\section{Tomoki TAKEDA, Masashi NAKAMURA, and Nagahiro HOSHI*}

\author{
Department of Applied Chemistry and Biotechnology, Graduate School of Engineering, \\ Chiba University, Yayoi-cho 1-33, Inage-ku, Chiba 263-8522, Japan \\ * Corresponding author: hoshi@faculty.chiba-u.jp
}

\begin{abstract}
Structural effects on the activity for the oxygen reduction reaction (ORR) have been studied on single crystal electrodes of Pt modified with six aromatic organic molecules (AOMs). The AOMs examined affect the ORR activity slightly. However, the activity of the sites uncovered by AOMs increases after the modification: the ORR activity of uncovered $\mathrm{Pt}(111)$ area after the modification of phthalocyanine is 2.5 times as high as that of bare $\mathrm{Pt}(111)$. $t$-BuTAP and iron (II) phthalocyanine also enhance the ORR on Pt(997). These facts show that adsorbed AOMs can enhance the ORR activity of the uncovered active sites on Pt electrodes.
\end{abstract}

(c) The Electrochemical Society of Japan, All rights reserved.

Keywords: Enhancement of the Activity for the Oxygen Reduction Reaction, Aromatic Organic Molecules, Pt Single Crystal Electrode

\section{Introduction}

Pt has the highest ORR activity among pure metals, and has been widely used for electrocatalysts such as polymer electrolyte fuel cells (PEFC). However, limitation of the natural resource, higher cost of $\mathrm{Pt}$ and higher overpotential of the oxygen reduction reaction (ORR) hinder the wide spread of fuel cells. It is indispensable to reduce the Pt loading by development of electrocatalysts that have higher activity for the ORR.

One of the strategies for the increase of the ORR activity is the structural regulation of the surface of a catalyst. Markovic et al. reported that the ORR activity on the low index plane of Pt increases as $\mathrm{Pt}(100)<\mathrm{Pt}(111)<\mathrm{Pt}(110)$ in $0.1 \mathrm{M} \mathrm{HClO}_{4}$, showing that the ORR activity depends on surface structure significantly. ${ }^{1}$

Feliu et al. studied the ORR on the high index planes of Pt. They found that introduction of step structures is a major factor improving the ORR activity using an exchange current density and a half wave potential as a measure of the ORR activity. ${ }^{2,3}$ We evaluated the ORR activity on the high index planes using a current density at $0.9 \mathrm{~V}$ (RHE) as a common measure, and found that (111) terrace edge enhances the ORR on Pt electrodes. ${ }^{4,5}$ DFT calculation attributed the high ORR activity of Pt single crystal electrodes with (111) terrace edge to the change of adsorbed water structure by the terrace edge. The change of water structure results in the hindrance of Pt oxides formation that deactivates the ORR. ${ }^{6}$

Modification of Pt electrodes by organic molecules can also change adsorbed water structure. The ORR activity of Pt nanoparticles is enhanced by the modification with octylamine (OA) and amine with a pyrene ring (PA) remarkably. ${ }^{7}$ We extended the study to the high index planes of $\mathrm{Pt}$, and found that $\mathrm{OA} / \mathrm{PA}$ enhances the ORR on Pt electrodes with more than 7 atomic rows of (111) terrace. $^{8}$

Modification by organic molecules can alter the vacancy density of the d-band, changing the adsorption force of $\mathrm{O}_{2} .{ }^{9}$ This effect can improve the ORR activity. Aromatic organic molecules (AOM) such as CoTPP and $\mathrm{C}_{60}$ affect the d-band vacancy due to the charge transfer from the adsorbed molecules. ${ }^{10,11}$ In this study, we have studied the ORR on single crystal electrodes of Pt modified with various AOMs as shown in Fig. 1(a). (a)

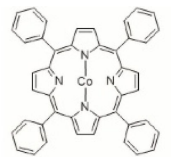
CoTPP

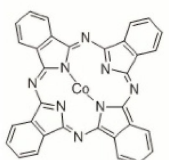

$\mathrm{CoPc}$

(b)

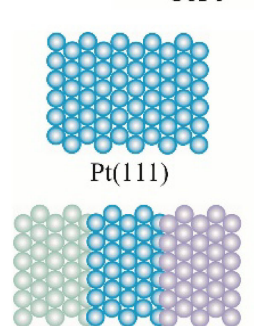

$\operatorname{Pt}(322)=5(111)-(100)$

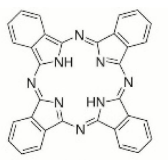

$\mathrm{Pc}$

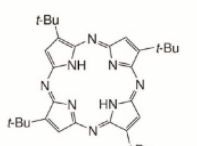

$t$-BuTAP

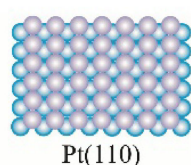

$\operatorname{Pt}(110)$

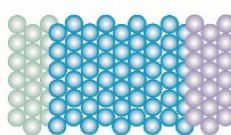

$\operatorname{Pt}(997)=9(111)-(111)$

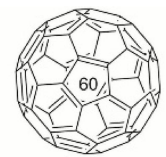

$\mathrm{C} 60$

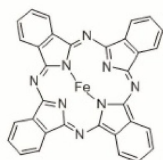

$\mathrm{FePc}$

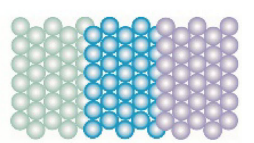

$\operatorname{Pt}(553)=5(111)-(111)$

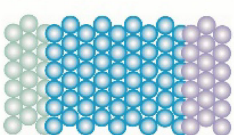

$\operatorname{Pt}(544)=9(111)-(100)$
Figure 1. (a) Aromatic organic molecules examined. (b) Hard sphere models of surfaces of Pt electrodes.

\section{Experimental}

CoTPP (5,10,15,20-tetraphenyl porphyrinato Cobalt(II)) and Pc (phthalocyanine) were purchased from Wako Pure Chemicals Co., Ltd. $\mathrm{C}_{60}$ (Fullerene), CoPc (Cobalt(II) phthalocyanine), $t$-BuTAP (2,7,12,17-Tetra-tert-butyl-5,10,15,20-tetraaza-21H,23H-porphine) and $\mathrm{FePc}$ (Iron(II) phthalocyanine) were purchased from SigmaAldrich Co., Ltd. Perchloric acid (ultrapure) and N,N-Dimethylformamide were purchased from Kanto Chemical Co., Inc.

A single crystal bead of Pt was prepared by Clavilier's method. ${ }^{12}$ The crystal was oriented using a reflection beam of a He-Ne laser ${ }^{13}$ and then mechanically polished with diamond slurries. Hard sphere models of the prepared electrodes are shown in Fig. 1(b). $\operatorname{Pt}(553)=5(111)-(111)$ and $\operatorname{Pt}(322)=5(111)-(100)$ have terrace widths that almost coincides with the size of AOMs examined. 
Terrace widths of $\operatorname{Pt}(997)=9(111)-(111)$ and $\operatorname{Pt}(544)=9(111)-$ (100) are larger than those of $\operatorname{Pt}(553)$ and $\operatorname{Pt}(322)$, but they accommodate only one AOM on the (111) terraces, giving more uncovered active sites.

A single crystal electrode of $\mathrm{Pt}$ was annealed in $\mathrm{H}_{2} / \mathrm{O}_{2}$ flame about $1300^{\circ} \mathrm{C}$ for the removal of distortions caused by the mechanical polishing, and then cooled to room temperature in $\mathrm{Ar} /$ $\mathrm{H}_{2}$ atmosphere $\left(\mathrm{Ar} / \mathrm{H}_{2}=8 / 2\right)$. The annealed surface was protected with ultrapure water before the modification of AOM.

Modification of AOM was carried out as follows.

1. DMF (N,N-Dimetylformamide) solution of AOM $(0.07 \mathrm{mM})$ was prepared.

2. An annealed single crystal electrode was immersed in the solution for $0.5-2.0 \mathrm{~h}$.

3. The electrode was rinsed with ultrapure water.

Electrolytic solution was prepared from ultrapure water treated with Milli-Q Advantage A10 (Millipore) and perchloric acid (ultrapure, Kanto Kagaku).

Linear sweep voltammograms of the ORR were measured in the hanging meniscus rotating disk electrode (HMRDE) configuration ${ }^{14}$ using an electrochemical analyzer (ALS $701 \mathrm{C}$ ) and a rotating ring disk electrode apparatus (BAS: RRDE-3). The potential was scanned from 0.05 to $1.0 \mathrm{~V}$ (RHE) at scanning rate $0.010 \mathrm{~V} \mathrm{~s}^{-1}$ and rotation rate $1600 \mathrm{rpm}$. We estimated the ORR activity using kinetic current density at $0.90 \mathrm{~V}$ (RHE) $j_{\mathrm{K}}$ calculated from the Koutecky-Levich equation. ${ }^{15,16}$

$$
\frac{1}{j}=\frac{1}{j_{K}}+\frac{1}{j_{L}}
$$

where $j, j_{\mathrm{K}}$ and $j_{\mathrm{L}}$ are the total current density, kinetic current density and limiting current density, respectively. All the potentials were referred to RHE.

\section{Results and Discussion}

Figure 2(a) shows voltammograms of single crystal electrodes of Pt before and after Pc modification as a representative. The single crystal surfaces were modified with Pc successfully because the charge of the adsorbed hydrogen region decreases. The charges of the oxide formation regions also decrease on $\operatorname{Pt}(111)$ and $\operatorname{Pt}(110)$ after the modification. Sharp redox peaks at $0.12 \mathrm{~V}$ (RHE) on $n(111)-(111)$ and $0.27 \mathrm{~V}$ (RHE) on $n(111)-(100)$ originate from the adsorption/desorption of hydrogen at (111) and (100) steps, respectively. ${ }^{17,18}$ Broad redox peaks between 0.05 and $0.4 \mathrm{~V}$ (RHE) are due to the adsorption/desorption of hydrogen at (111) terrace. Only the peaks due to the steps shrink after the modification, showing that Pc molecules are preferentially adsorbed at the steps. Coverage of $\mathrm{Pc}$ is estimated from the charge density of adsorbed hydrogen after the modification. Table 1 summarizes coverages of $\mathrm{Pc}$ at terraces and steps. Other organic aromatic molecules gave the similar results.

Figure 2(b) shows linear sweep voltammograms before and after the modification by Pc. Limiting current decreases after the modification on the high index planes, but the cause is unclear. The value of $j_{\mathrm{k}}$ at $0.90 \mathrm{~V}$ (RHE) is calculated using geometrical surface area of a single crystal electrode. Geometric surface area of a single crystal electrode gives real surface area because single crystal surface of Pt is atomically flat. Figure 3(a) shows the values of $j_{\mathrm{k}}$ and the ORR activity normalized to that of bare Pt single crystal electrode. Surprisingly, all the AOMs except $\mathrm{Pc}$ and $\mathrm{C}_{60}$ enhance the ORR activity on $\operatorname{Pt}(111)$ slightly, although nearly $40 \%$ of the surface area is covered by the AOMs. On the other hand, the ORR on the other surfaces are deactivated by AOMs except the cases of $t$-BuTAP on $\operatorname{Pt}(110)$ and $\operatorname{Pt}(997)$.

The slight enhancement of the ORR activity after the modification indicates that the ORR activity of uncovered active

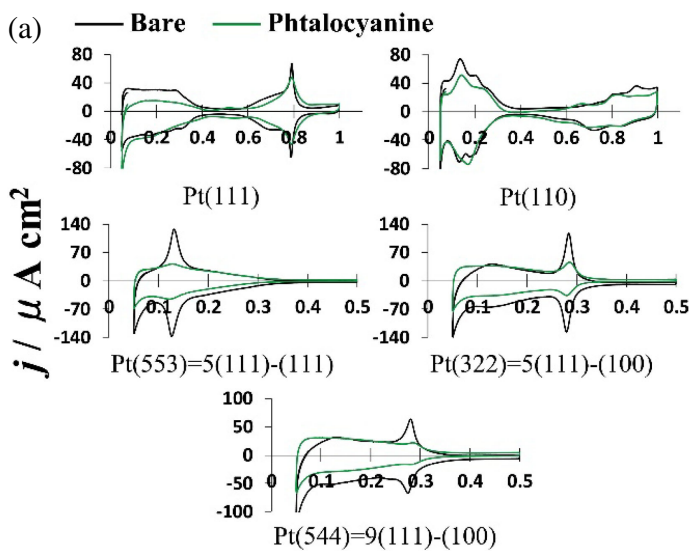

$E$ / V (vs.RHE)

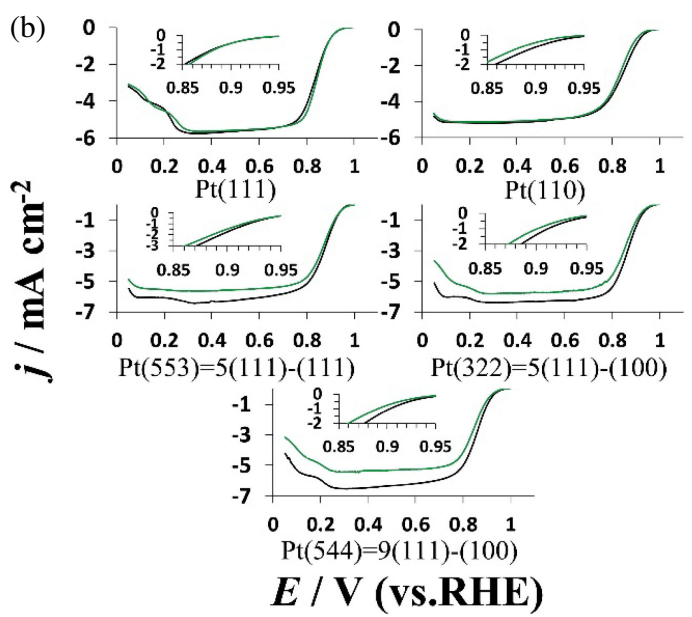

Figure 2. (a) Cyclic voltammograms of $\mathrm{Pt}$ electrodes before and after the modification with $\mathrm{Pc}$ in $0.1 \mathrm{M} \mathrm{HClO}_{4}$ saturated with $\mathrm{Ar}$ (Scanning rate: $0.050 \mathrm{~V} \mathrm{~s}^{-1}$ ). (b) Linear sweep voltammograms of $\mathrm{Pt}$ electrodes before and after the modification with $\mathrm{Pc}$ in $0.1 \mathrm{M} \mathrm{HClO}_{4}$ saturated with $\mathrm{O}_{2}$ (Scanning rate: $0.010 \mathrm{~V} \mathrm{~s}^{-1}$, Scanning direction: Positive, Rotation rate: $1600 \mathrm{rpm}$ ).

Table 1. Coverage of Pc at terrace $\left(\theta_{\text {terrace }}\right)$ and step $\left(\theta_{\text {step }}\right)$.

\begin{tabular}{ccc}
\hline & $\theta_{\text {terrace }}$ & $\theta_{\text {step }}$ \\
\hline $\operatorname{Pt}(111)$ & 0.61 & - \\
$\operatorname{Pt}(110)$ & - & 0.12 \\
$\operatorname{Pt}(553)$ & 0.12 & 0.86 \\
$\operatorname{Pt}(322)$ & 0.02 & 0.54 \\
$\operatorname{Pt}(544)$ & 0.24 & 0.88 \\
\hline
\end{tabular}

sites is enhanced compared with a Pt electrode without modification. We calculate the ORR activity of uncovered active sites using the electrochemically active surface area (ECSA) after the modification. We calculated ECSA by the division of the charge of hydrogen desorption $(0.05-0.4 \mathrm{~V})$ by $210 \mathrm{mC} \mathrm{cm}^{-2}{ }^{19}$ Figure $3(\mathrm{~b})$ shows the normalized ORR activity of uncovered active sites. The ORR activity of uncovered active sites is enhanced on $\operatorname{Pt}(111)$ by the modification of all the AOMs. Pc enhances the ORR on $\mathrm{Pt}(111)$ most remarkably: the ORR activity of the uncovered active sites on Pc modified $\mathrm{Pt}(111)$ is 2.5 times as high as that of bare $\mathrm{Pt}(111)$. The following combinations also enhance the ORR activity of uncovered active sites after the modification slightly: (CoTPP, Pc, CoPc)/ $\mathrm{Pt}(110),(\mathrm{Pc}, \mathrm{CoPc}) / \mathrm{Pt}(553),(t$-BuTAP, FePc)/Pt(322), $(t$-BuTAP, $\mathrm{FePc}) / \mathrm{Pt}(997)$ and $\mathrm{FePc} / \mathrm{Pt}(544)$. The ORR activity of $\mathrm{FePc}$ on glassy carbon is negligible compared with those of FePc covered 

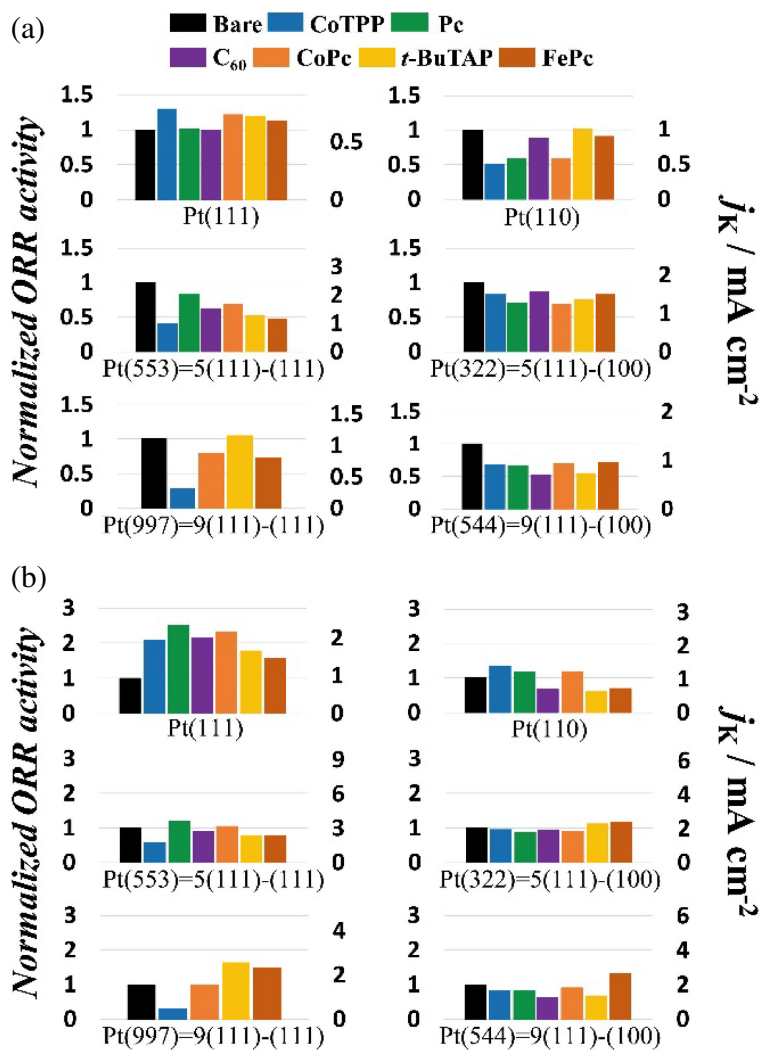

Figure 3. (a) The ORR activity after the modification with aromatic organic molecules (evaluated by geometrical surface area) normalized to that before the modification. (b) The ORR activity of the sites uncovered by aromatic molecule normalized to that before the modification

single crystal electrodes of Pt at $0.9 \mathrm{~V}$ (RHE). ${ }^{20,21}$ In ultra-high vacuum, electron transfer is induced between $\mathrm{FePc}$ and a mental substrate, resulting in a change of electronic state of FePc. ${ }^{22}$ The same phenomenon might occur in electrochemical environments, and the ORR activity of FePc might be enhanced. However, we cannot evaluate the ORR activity of FePc adsorbed on Pt electrodes because we cannot increase the coverage of FePc more than 0.4. Thus we assume that the ORR activities of AOMs are negligible at $0.90 \mathrm{~V}$ (RHE) on Pt electrodes. Based on this assumption, the ORR activity of $\mathrm{AOM} / \mathrm{Pt}(h k l)$ originates from Pt surface uncovered by AOM.

Control experiment was done after a Pt single crystal electrode was immersed in DMF solution without AOM. The charge of the adsorbed hydrogen region decreased slightly, but the change was small compared with that after AOMs modification. The ORR activity was slightly decreased after the modification of DMF. These facts show that DMF has no effect on the enhancement of the ORR activity.

There are several reasons for the improvement of the ORR activity. The first one is the shift of the d-band center by the electron attractive force of AOMs. The second one is the change of the adsorbed water structure due to the modification that prevents $\mathrm{Pt}$ oxide formation. However, modification of $\mathrm{C}_{60}$ does not improve the ORR activity on all the surfaces, although $\mathrm{C}_{60}$ has strong electron attractive property. ${ }^{11}$ Shift of d-band center prevents the formation of Pt oxides that block the ORR. AOMs inhibit the Pt oxides formation as shown in the voltammograms of $\mathrm{Pt}(111)$ in Fig. 2(a). However, the decrease of Pt oxide formation is too small to cause the remarkable enhancement of the ORR activity of the surface uncovered by AOMs. IR spectroscopy shows that ice-like water structure is formed on Pt single crystal electrodes after the modification of amines. The ice-like structure may stabilize water, resulting in the prevention of the formation of $\mathrm{Pt}$ oxides that block the ORR. ${ }^{8} \operatorname{Pt}(111)$ has a wider (111) terrace structure that is appropriate for the ice-like structure (hexamer) formation. Therefore, the ORR activity may be enhanced most significantly. It is possible that ice-like structure formation is one of the factors of the enhancement of the ORR.

Study of the vibrational spectroscopy of the adsorbed water is necessary to reveal the enhancement mechanism, and is now on progress in our laboratory.

\section{Conclusion}

Adsorbed aromatic organic molecules (AOMs) enhance the ORR activity of uncovered active sites on $\operatorname{Pt}(111)$ : the ORR activity of the uncovered active sites on phthalocyanine modified $\mathrm{Pt}(111)$ is 2.5 times as high as that of bare $\operatorname{Pt}(111)$. The following combinations also enhance the ORR activity of uncovered active sites after the modification slightly: $(5,10,15,20$-tetraphenyl porphyrinato Cobalt(II), phthalocyanine, Cobalt(II) phthalocyanine)/Pt(110), (phthalocyanine, Cobalt(II) phthalocyanine)/Pt(553), (2,7,12,17Tetra-tert-butyl-5,10,15,20-tetraaza-21H,23H-porphine, iron(II) phthalocyanine $) / \operatorname{Pt}(322), \quad(2,7,12,17-$ Tetra-tert-butyl-5,10,15,20tetraaza-21H,23H-porphine, iron(II) phthalocyanine)/Pt(997) and iron(II) phthalocyanine/Pt(544).

\section{Acknowledgment}

This work was supported by New Energy Development Organization (NEDO).

\section{References}

1. N. M. Markovic, R. R. Adzić, B. D. Cahan, and E. B. Yeager, J. Electroanal. Chem., 377, 249 (1994).

2. M. D. Maciá, J. M. Campiña, E. Herrero, and J. M. Feliu, J. Electroanal. Chem., 564, 141 (2004).

3. A. Kuzume, E. Herrero, and J. M. Feliu, J. Electroanal. Chem., 599, 333 (2007).

4. A. Hitotsuyanagi, M. Nakamura, and N. Hoshi, Electrochim. Acta, 82, 512 (2012).

5. N. Hoshi, M. Nakamura, and A. Hitotsuyanagi, Electrochim. Acta, 112, 899 (2013).

6. R. Jinnouchi, K. Kodama, and Y. Moromoto, J. Electroanal. Chem., 716, 31 (2014).

7. K. Miyabayashi, H. Nishihara, and M. Miyake, Langmuir, 30, 2936 (2014).

8. K. Saikawa, M. Nakamura, and N. Hoshi, Electrochem. Commun., 87, 5 (2018).

9. S. Mukerjee, S. Srinivasan, and M. P. Soriaga, J. Electrochem. Soc., 142, 1409 (1995).

10. K. Suto, S. Yoshimoto, and K. Itaya, Langmuir, 22, 10766 (2006).

11. C.-T. Tzeng, W.-T. Lo, J.-Y. Yuh, R.-Y. Chu, and K.-D. Tsuei, Phys. Rev. B, 61, $2263(2000)$

12. J. Clavilier, R. Faure, G. Guinet, and R. Durand, J. Electroanal. Chem., 107, 205 (1980).

13. N. Furuya and S. Koide, Surf. Sci., 220, 18 (1989).

14. B. D. Cahan and H. M. Villullas, J. Electroanal. Chem., 307, 263 (1991).

15. H. M. Villullas and M. Lopez Teijelo, J. Electroanal. Chem., 384, 25 (1995)

16. H. M. Villullas and M. Lopez Teijelo, J. Electroanal. Chem., 385, 39 (1995).

17. J. Clavilier, K. El Achi, and A. Rodes, Chem. Phys., 141, 1 (1990).

18. J. Clavilier, D. Armond, S. G. Sun, and M. Petit, J. Electroanal. Chem., 205, 267 (1986).

19. H. A. Gasteiger, S. S. Kocha, B. Sompalli, and F. T. Wagner, Appl. Catal., B, 56, 9 (2005).

20. S. Baranton, C. Coutanceau, C. Roux, F. Hahn, and J.-M. Leger, J. Electroanal. Chem., 577, 223 (2005).

21. M. Inamoto, H. Kurihara, and T. Yajima, Electrochemistry, 85, 469 (2017).

22. J. Uihlein, H. Peisert, H. Adler, M. Glaser, M. Polek, R. Ovsyannikov, and T. Chassé, J. Phys. Chem. C, 118, 10106 (2014). 